\title{
Analysis of dip coating processing parameters by double optical monitoring
}

\author{
Flavio Horowitz* and Alexandre F. Michels \\ Instituto de Física, Universidade Federal do Rio Grande do Sul, Campus do Vale CP15051, \\ 91501-970 Porto Alegre, RS, Brazil \\ *Corresponding author: flavio.horowitz@ufrgs.br
}

Received 30 August 2007; accepted 26 October 2007;

posted 7 November 2007 (Doc. ID 87033); published 20 December 2007

\begin{abstract}
Double optical monitoring is applied to determine the influence of main process parameters on the formation of sulfated zirconia and self-assembled mesoporous silica solgel films by dip coating. In addition, we analyze, for the first time to the best of our knowledge, the influence of withdrawal speed, temperature, and relative humidity on refractive-index and physical thickness variations (uncertainties of \pm 0.005 and $\pm 7 \mathrm{~nm}$ ) during the process. Results provide insight into controlled production of single and multilayer films from complex fluids by dip coating. (C) 2008 Optical Society of America

OCIS codes: $310.3840,310.6860,260.3160$.
\end{abstract}

\section{Introduction}

Dip coating, a technique whose basic configuration requires unsophisticated equipment, has been advantageously used in the production of films on nonplanar or long-dimension substrates for decades [1,2], with the measurement of film thicknesses taken by capacitive methods. These are influenced by other parameters such as substrate or belt thickness variations and present a typical uncertainty of $\pm 2 \mu \mathrm{m}$ [3]. Optical monitoring, on the other hand, has been used through transmission or reflection interferometry for solid thin films under vacuum deposition since the 1950s [4], whereas its application to spin coating of liquid films is more recent [5].

With regard to optical monitoring of dip coating, first experiments involved nonvolatile, Newtonian mineral oils with a constant refractive index and viscosity during the entire process. Once known the constant refractive index by Abbe refractometry, interferometric monitoring provided temporal evolution of optical thickness, thus allowing determination of physical thickness evolution in real time. Predictions of a simple theoretical model of drainage for

0003-6935/08/130C $185-4 \$ 15.00 / 0$

(C) 2008 Optical Society of America
Newtonian fluids could then be successfully tested $[6,7]$.

For more complex fluids in which both refractive index and physical thickness vary during the process, a combination of polarimetric and interferometric (double optical) monitoring was introduced, and sensitivity of the method to changes in film-formation stages was demonstrated [8]. In the drainage stage, dominated by mass loss and use of a solgel sulfated zirconia colloid (SZC), the film was shown to be practically unaffected by evaporation and to flow as a Newtonian liquid [9]. Agreement with the theoretical prediction of physical thickness variation on time, proportional to $t^{-1 / 2}$, was attained with an accuracy much higher than in the traditional capacitive method (uncertainty of $\pm 0.007 \mu \mathrm{m}$ ).

From the accuracy and consistency previously shown by double optical monitoring, the main goal of our research was to evaluate in detail the influence of main process parameters (withdrawal speed, temperature, and relative humidity) in liquid film evolution, with varying refractive indices, during the production of SZC and self-assembled mesoporous silica solgel films.

\section{Experimental}

The sols used in this study, SZC and SBA-15 (a sol for mesoporous silica films), were previously produced 


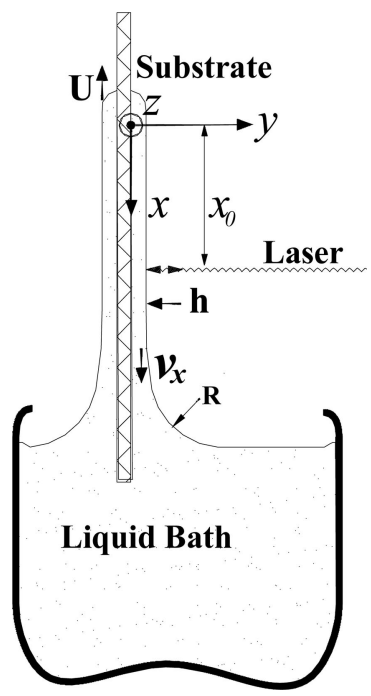

Fig. 1. Illustration of the dip coating batch process with laser probing.

according to Refs. [10] and [11], respectively. The optical experiment is essentially based on measurement of a laser beam (wavelength $\lambda=660 \mathrm{~nm}$ ) after normal reflection by a liquid film flowing on a glass or silicon substrate. The reflected light is compared with a reference signal and later processed by a lock-in amplifier and a computer with an analog-to-digital converter at a rate of $3.5 \mathrm{kHz}$. The spot under measurement corresponds to a fixed value in the vertical $x$ axis $\left(x_{0}\right.$ in Fig. 1). A more detailed description can be found in Ref. [8].

Typical interferograms, as obtained during dip coating of a SZC film, are illustrated in Fig. 2, where each interval between successive extremes corresponds to an optical thickness variation of $\lambda / 4$. This procedure, combined with the refractive-index values provided by polarimetric monitoring [8], allows determination of physical thickness variation values, as shown in the resulting figures that follow.
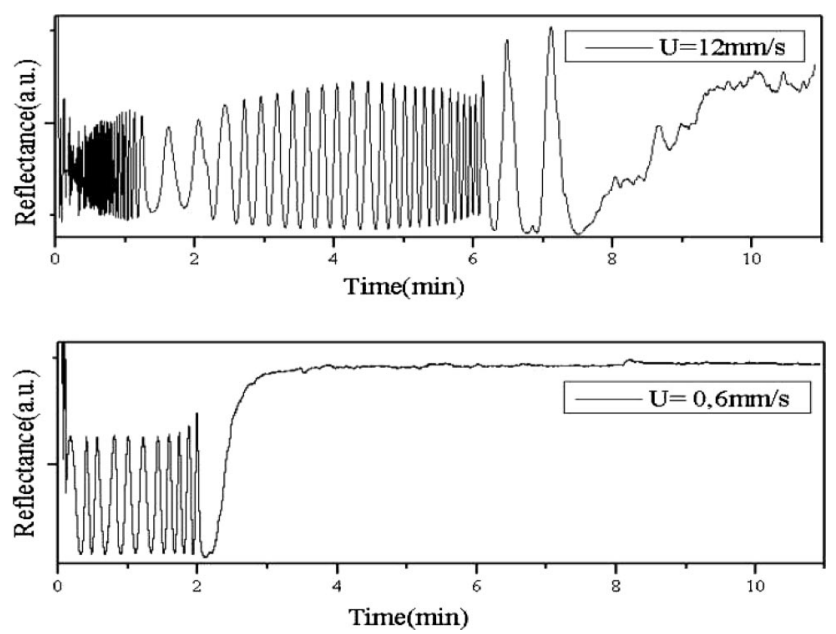

Fig. 2. Reflectance temporal evolution of SZC films during dip coating at different withdrawal speeds (U).

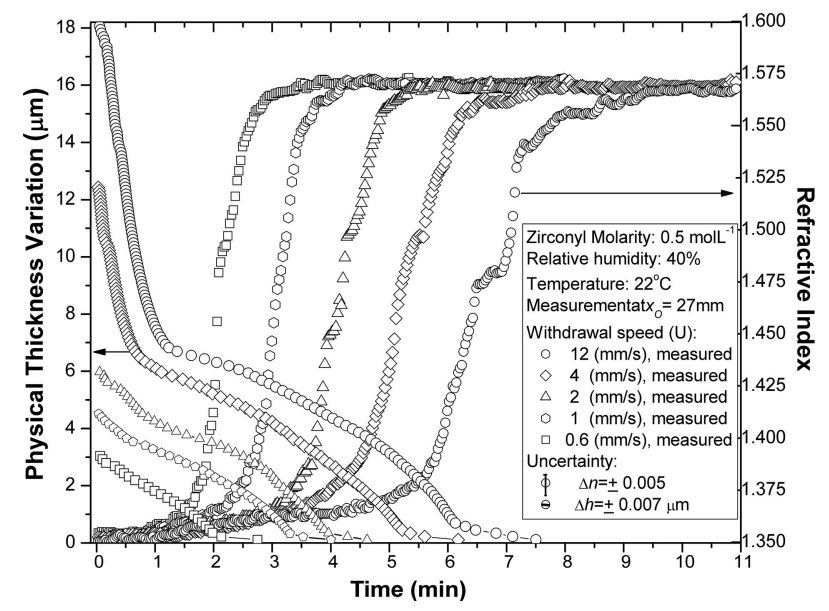

Fig. 3. Temporal evolution of physical thickness and refractive index during dip coating of SZC at different withdrawal speeds.

\section{Results and Discussion}

Liquid film evolution for a given composition (SZC or SBA-15) with varying refractive indices is evaluated during dip coating with regard to the influence of main process parameters, namely, withdrawal speed, temperature, and relative humidity. Different withdrawal speeds are used for SZC and SBA-15 films in Figs. 3 and 4 . As the withdrawal speed increases, so does the mass drain in the same time interval. On the other hand, a larger film thickness adheres to the substrate for a faster withdrawal speed, as indicated by the equation that describes physical thickness evolution during the drainage stage $[1,6]$ :

$$
h=C \sqrt{\frac{\mu}{g \rho} \frac{x}{t}},
$$

where $U=x / t$ corresponds to the withdrawal speed for an observer in the laboratory reference frame and $C=f\left(C_{a}\right)$, i.e., a function of the capillary number

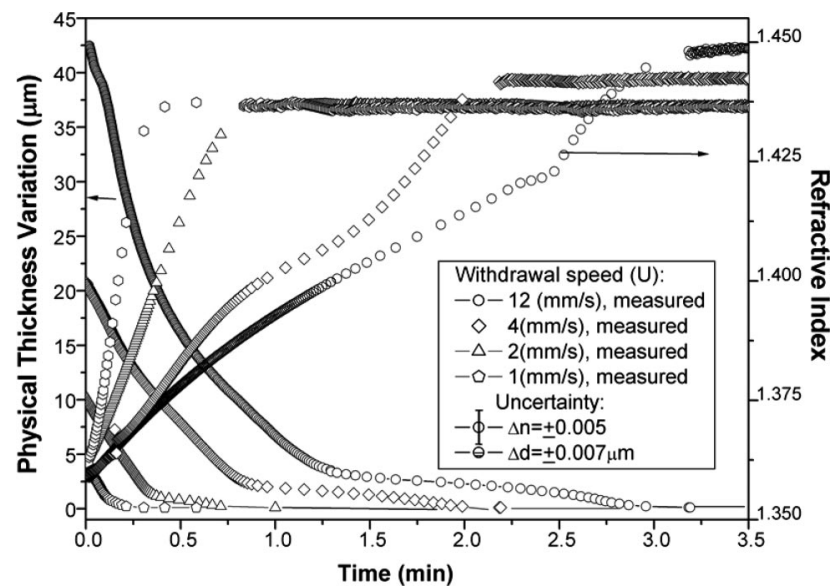

Fig. 4. Temporal evolution of physical thickness and refractive index during dip coating of SBA-15 solgel at different withdrawal speeds. 
$C a \equiv \mu U / \sigma$ [3], where $\mu$ is the coefficient of viscosity, $\sigma$ is the surface tension, and $\rho g$ is the weight per unit volume.

The two observations above are consistent, since a thicker film allows faster flow in its most external layers. Also, an increase in film thickness implies an increase in the time required for the abrupt change in refractive index that corresponds to gelation. This is observed in the formation of both SZC and SBA-15 films, as seen in Figs. 3 and 4. Note also that, although refractive-index accuracy is within \pm 0.005 , the precision shown is around \pm 0.001 (see the reproducibility in a large number of measurements at each plateau). However, all the refractive-index curves reach the same plateau (at different times) in Fig. 3, whereas slightly different plateaus are reached by the refractive-index curves in Fig. 4. This may be attributed to the self-assembly process that occurs with SBA-15 during dip coating but not with SZC. In the SBA-15 evolution, it seems that the higher time required for gelation at higher speeds allows for more structural ordering and densification, thus leading to a higher postgelation refractive-index value.

At constant withdrawal speed, as seen in Fig. 5, the increase in processing temperature promotes an earlier outcome of the refractive-index shoulder as well as higher-index values after gelation in connection with solvent loss and higher densification. These tend to increase sol viscosity, opposing its tendency to decrease with an increase in temperature, thus resulting in a small dependence of physical thickness variation on temperature in the observed time interval.

In Fig. 6, an increase in relative humidity of the surrounding atmosphere, which affects solvent evaporation, has a pronounced influence on the formation of ZSC films, delaying the start of gelation as well as decreasing the postgelation refractive index (to the first decimal place). Relative humidity, on the other hand, has little effect on physical thickness variation, probably because of the opposing influence of viscosity increase and mass loss by solvent evaporation.

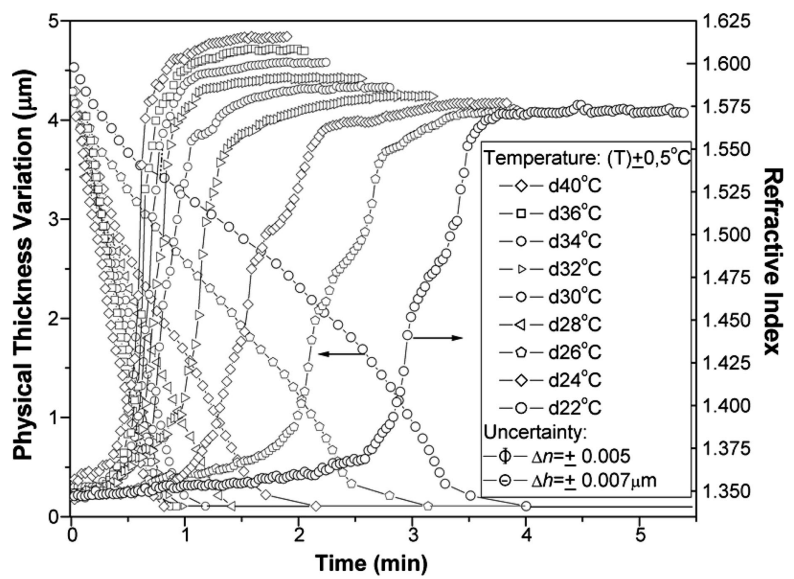

Fig. 5. Temporal evolution of physical thickness and refractive index during dip coating of SZC at different temperatures.

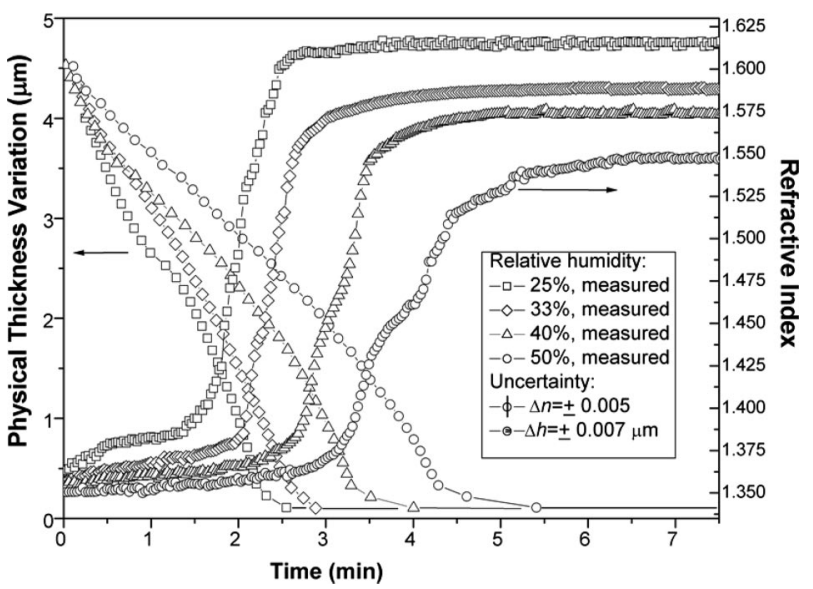

Fig. 6. Temporal evolution of physical thickness and refractive index during dip coating of SZC at different relative humidities.

\section{Concluding Remarks}

Double optical monitoring was applied during dip coating with SZC and SBA-15 fluids to determine temporal evolution of both physical thickness and refractive index. Corresponding accuracies are within $\pm 0.007 \mu \mathrm{m}$ and \pm 0.005 , respectively, whereas precision is even better (around \pm 0.001 in refractive index). This allowed evaluation of how liquid film formation is influenced by withdrawal speed, temperature, and relative humidity in real time. For both SZC and SBA-15 films, an increase in processing temperature or relative humidity has little influence on physical thickness variation, probably because of the interplay of opposing influences of viscosity and of mass loss by solvent evaporation. Also, an increase in processing temperature promotes an earlier outcome of the refractive-index shoulder, as well as higherindex values after gelation. However, opposite effects (but much more pronounced on postgelation refractive index) are produced by an increase in relative humidity. On the other hand, higher withdrawal speeds have no significant effect on SZC postgelation refractive index but they do have a noticeable influence on that of SBA-15. This seems to be due to structural ordering and densification by selfassembly in the latter during dip coating, which is a point that deserves further investigation.

These double optical process monitoring results in real time not only corroborate well-known postprocess observations on physical thickness but also provide insight for controlled production of single and multilayer films from complex fluids. As to the measured refractive-index changes during stages that follow mass drainage, quantitative analysis with regard to structural and compositional film variations is currently under study.

This research was partially supported by Conselho Nacional de Desenvolvimento Cientifico e Tecnologico under the Recursos Humanos para Areas Estrategicas, Empresa Brasileira de Compressores innovation project agreement with special acknowledgment to researcher Fabian Fagotti. We are also 
grateful to our colleagues Celso Santilli at Universidade Estadual Paulista and Marcia Fantini at Universidade de Sao Paulo for providing us with the SZC and SBA-15 sols, respectively.

\section{References}

1. L. E. Scriven, "Physics and applications of dip coating and spin coating," Mater. Res. Soc. 121, 717-729 (1988).

2. P. R. Schunk, A. J. Hurd, and C. J. Brinker, "Free-meniscus coating processes," in Liquid Film Coating, S. F. Kistler and P. M. Schweizer, eds. (Chapman \& Hall, 1997), pp. 673-708.

3. R. P. Spiers, C. V. Subabaraman, and W. L. Wilkinson, "Free coating of a Newtonian liquid onto a vertical surface," Chem. Eng. Sci. 29, 389-396 (1973).

4. H. A. Macleod, Thin-Film Optical Filters, 2nd ed. (Macmillan, 1986).

5. F. Horowitz, E. M. Yeatman, E. Dawnay, A. Fardad, and M. Green, "Real-time optical monitoring of spin coating," J. Phys (Paris) III 3, 2059-2063 (1993).
6. A. F. Michels, T. Menegotto, and F. Horowitz, "Interferometric monitoring of dip coating," Appl. Opt. 43, 820-823 (2004).

7. A. F. Michels, T. Menegotto, and F. Horowitz, "Optically monitored dip coating as a contactless viscometry method for liquid films," Appl. Opt. 44, 912-915 (2005).

8. A. F. Michels, T. Menegotto, H. P. Grieneisen, M. B. Susin, and F. Horowitz, "Double optical monitoring of dip coating with a time-varying refractive index," Appl. Opt. 45, 1491-1494 (2006).

9. A. F. Michels, T. Menegotto, C. V. Santilli, and F. Horowitz, "Temporal evolution of sulfated zirconia sol-gel during dip coating: analysis of mass drainage with time-varying refractive index," J. Non-Cryst. Solids 352, 5362-5367 (2006).

10. L. A. Chiavacci, S. H. Pulcinelli, and C. V. Santilli, "Structural and phenomenological characterization of the thermoreversible sol-gel transition of a zirconyl aqueous precursor modified by sulfuric acid," Chem. Mater. 10, 986-993 (1998).

11. R. M. Grudzien, B. E. Grabicka, and M. Jaroniec, "Effective method for removal of polymeric template from SBA-16 silica combining extraction and temperature-controlled calcinations," J. Mater. Chem 16, 819-823 (2006). 